\title{
Nonlinear Lamb wave mixing for assessing localized deformation during creep
}

\author{
Avijit Kr Metya ${ }^{\text {a,c, }{ }^{*}, \text { Soumitra Tarafder }}{ }^{\mathrm{b}}$, Krishnan Balasubramaniam ${ }^{\mathrm{c}}$ \\ a AMP- Division, CSIR- National Metallurgical Laboratory, Jamshedpur 831007, India \\ ${ }^{\mathrm{b}}$ MTE- Division, CSIR- National Metallurgical Laboratory, Jamshedpur 831007, India \\ ${ }^{\mathrm{c}}$ CNDE, Mechanical Engineering Department, Indian Institute of Technology Madras, Chennai 600036, India
}

\section{A R T I C L E I N F O}

Keywords:

Lamb wave mixing

Nonlinear ultrasonic

Localized deformation

Modified P91 steel

\begin{abstract}
A B S T R A C T
Nonlinear ultrasonic is known to be a promising technique to characterize the microstructural degradation in engineering materials. This work demonstrates the use of nonlinear Lamb wave mixing technique to assess the localized deformation in modified $9 \mathrm{Cr}-1 \mathrm{Mo}$ steel during creep. Two Lamb wave modes of different frequencies $\left(\omega_{1} \& \omega_{2}\right)$ are allowed to mix within the material under certain resonant condition to generate third type of harmonic waves of frequencies $\left(\omega_{1} \pm \omega_{2}\right)$. This new generated wave carries the information of material nonlinearity from the mixing site and independent of the other extraneous nonlinear factors. Amplitude of the generated third wave depends on the third order elastic constants of the material. This study reveals that nonlinear Lamb wave mixing technique could be used to assess the localized deformation much prior to its failure.
\end{abstract}

\section{Introduction}

Nonlinear ultrasonic (NLU) technique has shown to be a potential NDE tool due to its ability to characterize damage much prior to the failure. It uses the interrogation signals at frequencies other than the excitation frequency to detect changes in structural integrity and characterization of material degradation [1]. It relies on the nonlinear stress-strain behavior or nonlinear Hooke's law that is dominated by higher order elastic constants in isotropic elastic solid. Initial sinusoidal elastic wave gets distorted and generates higher order harmonics in presence of nonlinearity within the materials. This NLU technique assesses the average nonlinearities over the region between transmitter and receiver; so, spatial resolution is limited. But the major problem in NLU technique/harmonic generation is to distinguish the causes of nonlinearity. Because, there are multiple extraneous sources that generate higher harmonics beside material nonlinearity and these may be the instrumentation of the measurement system, coupling media or other external factors [2]. So, the measured nonlinearity includes the material nonlinearity along with the nonlinearities generated from extraneous sources.

Attempts have been made to characterize the materials using wave mixing technique to overcome the above mentioned limitations. Although theoretical study on nonlinear wave mixing has been started since 1960s [3-8]. Croxford et al. reported the use of non-collinear mixing technique to the ultrasonic measurement of material nonlinearity to assess plasticity and fatigue damage [9]. They showed that this technique is potentially more attractive for assessing material state than other nonlinear ultrasonic techniques. Demcenko et al. showed the sensitivity of non-collinear wave mixing for nonlinear ultrasonic detection of physical ageing in PVC [10]. Physical ageing state in PVC was characterized using both linear ultrasonic measurement technique and the non-collinear ultrasonic wave mixing technique. Works have also been done of using collinear wave mixing technique for nonlinear ultrasonic measurement to characterize the material damage conditions $[11,12]$. All these studies reported the use of bulk wave mixing to characterize the state of the damage in materials but little research has been done on Lamb wave mixing to assess the material degradation. Initial feasibility study of Lamb wave mixing has been performed by D J lee et al. [13]. Some aspects of guided wave mixing have also been discussed by C J Lissenden et al. [14]. Very recently J Jingpin et al. used the nonlinear Lamb wave mixing technique for micro-crack detection in plates [15]. But no work has been performed to use the Lamb wave mixing technique to assess the localized deformation in materials. In this work, it has been demonstrated the use of nonlinear Lamb wave mixing technique to detect the location of maximum localized deformation in material during creep much prior to its failure.

\footnotetext{
* Corresponding author. AMP- Division, CSIR- National Metallurgical Laboratory, Jamshedpur 831007, India.

E-mail addresses: avijit@nmlindia.org, avijit353@gmail.com (A.K. Metya).
} 


\section{Mixing of lamb waves}

\subsection{Lamb wave propagation and mixing}

The phenomenon of elastic waves in thin plates in isotropic, stress free, linear and homogeneous media is given as Rayleigh- Lamb frequency relations [16].

$\frac{\tan (q h)}{\tan (p h)}=-\left(\frac{4 k^{2} p q}{\left(q^{2}-k^{2}\right)^{2}}\right)^{ \pm 1}$

where, the exponent \pm 1 indicates the symmetric and anti-symmetric modes respectively and $p^{2}=\frac{\omega^{2}}{c_{L}^{2}}-k^{2}$ and $q^{2}=\frac{\omega^{2}}{c_{T}^{2}}-k^{2}, h$ is the plate thickness, $\omega$ is the angulay frequency, $k$ is the wave number, $c_{L}$ and $c_{T}$ are the longitudinal and transverse waves velocity respectively.

So, it can be seen from eqn. (1) that the propagation of Lamb waves is dispersive in nature and having multi-modal characteristics.

Now, the simple extension from linear dynamic elasticity to nonlinear equation requires the introduction of third order elastic constants-l, $m$, and $n$ (Murnaghan's constants considering the materials to be hyperelastic [17]). The nonlinear equation of motion for the displacement field $\mathbf{u}$ for an isotropic elastic material without any viscous dissipation takes the form [18].

$\rho \frac{\partial^{2} u_{i}}{\partial t^{2}}-\mu \frac{\partial^{2} u_{i}}{\partial x_{k}^{2}}-(\lambda+\mu) \frac{\partial^{2} u_{k}}{\partial x_{k} \partial x_{i}}=F_{i}$

where, $F_{i}$ contains all the nonlinear terms.

$$
\begin{aligned}
F_{i}= & {\left[\mu+\frac{n}{4}\right]\left[\frac{\partial^{2} u_{s}}{\partial x_{k}^{2}} \frac{\partial u_{s}}{\partial x_{i}}+\frac{\partial^{2} u_{s}}{\partial x_{k}^{2}} \frac{\partial u_{i}}{\partial x_{s}}+2 \frac{\partial^{2} u_{i}}{\partial x_{s} \partial x_{k}}\right]+\left[m-\frac{n}{4}\right]\left[\frac{\partial^{2} u_{s}}{\partial x_{i} \partial x_{k}} \frac{\partial u_{s}}{\partial x_{k}}\right.} \\
& \left.+\frac{\partial^{2} u_{k}}{\partial x_{s} \partial x_{k}} \frac{\partial u_{i}}{\partial x_{s}}\right]+\left[\lambda+m-\frac{n}{2}\right] \frac{\partial^{2} u_{i}}{\partial x_{k}^{2}} \frac{\partial u_{s}}{\partial x_{s}}+\left[m-\frac{n}{4}\right]\left[\frac{\partial^{2} u_{k}}{\partial x_{s} \partial x_{k}} \frac{\partial u_{s}}{\partial x_{i}}\right. \\
& \left.+\frac{\partial^{2} u_{s}}{\partial x_{i} \partial x_{k}} \frac{\partial u_{k}}{\partial x_{s}}\right]+\left[2 l-m+\frac{n}{2}\right] \frac{\partial^{2} u_{k}}{\partial x_{i} \partial x_{k}} \frac{\partial u_{s}}{\partial x_{s}}
\end{aligned}
$$

Rectangular co-ordinates have been used in terms of $x_{1}, x_{2}$, and $x_{3}$; subscripts appearing twice in a single term indicate summation over 1,2 , and 3.

Considering the elastic wave propagating along $x_{1}$ direction with a particle displacement along $x_{1} \& x_{2}$ direction, eqn. (2) can be expressed in scalar Cartesian notation as:

$\frac{\partial^{2} u_{1}}{\partial t^{2}}-c_{p}^{2} \frac{\partial^{2} u_{1}}{\partial x^{2}}=c_{p}^{2} \beta \frac{\partial}{\partial x}\left(\frac{\partial u_{1}}{\partial x}\right)^{2}+c_{p}^{2} \gamma \frac{\partial}{\partial x}\left(\frac{\partial u_{2}}{\partial x}\right)^{2}$

$\frac{\partial^{2} u_{2}}{\partial t^{2}}-c_{s}^{2} \frac{\partial^{2} u_{2}}{\partial x^{2}}=2 c_{p}^{2} \gamma \frac{\partial}{\partial x}\left(\frac{\partial u_{1}}{\partial x} \frac{\partial u_{2}}{\partial x}\right)$

where,

$c_{p}^{2}=\frac{\lambda+2 \mu}{\rho}, c_{s}^{2}=\frac{\mu}{\rho}, \beta=\frac{3(\lambda+2 \mu)+2(2 m+l)}{2(\lambda+2 \mu)}, \gamma=\frac{\lambda+2 \mu+m}{2(\lambda+2 \mu)}$

$\lambda$ and $\mu$ are Lame's constants.

These types of nonlinear boundary value problems [eqn. - (4) \& (5)] can be solved by perturbation method where the solution $(\mathbf{u})$ is expressed as the sum of two terms

$\mathbf{u}=\mathbf{u}^{(0)}+\mathbf{u}^{(1)}$

where $\mathbf{u}^{(0)}$ is the primary solution and $\mathbf{u}^{(1)}$ is the secondary solution. The solution $\mathbf{u}^{(2)}$ is the perturbation solution due to nonlinearity and it is assumed

$\left|\mathbf{u}^{(0)}\right| \gg\left|\mathbf{u}^{(1)}\right|$
Now during wave mixing experiment, if the excitation signal consists of two sinusoidal signals of different frequencies, the primary solution can be expressed as

$u^{(0)}(x, t)=A\left(x_{2}\right) e^{i\left(\omega_{1} t_{1}-k_{1} x\right)}+B\left(x_{2}\right) e^{i\left[\omega_{2}(t-\Delta \mathrm{T})-k_{2} x\right]}$

where, $k_{1}, \omega_{1}, A \& B$ are the wave number, angular frequency and amplitudes of the two sinusoidal waves respectively and $\Delta \mathrm{T}$ is the time delay that has been given to one of the fundamental waves in the exciting signal based on the group velocities of the Lamb wave modes for spatial scanning. Substituting eqn. (6) into nonlinear part of eqns. (4) and (5) results in terms containing the exponential functions

$e^{i\left[\left(\omega_{1} \pm \omega_{2}\right) t-\left(k_{1} \pm k_{2}\right) x\right]}, e^{-i\left[\left(\omega_{1} \pm \omega_{2}\right) t-\left(k_{1} \pm k_{2}\right) x\right]}, e^{i\left[2\left(\omega_{1} t-k_{1} x\right)\right]}, e^{i\left[2\left(\omega_{2} t-k_{2} x\right)\right]}$ for $\omega_{1}>\omega_{2}$

These terms correspond to possible second order harmonic waves at $\left(\omega_{1}+\omega_{2}, k_{1}+k_{2}\right),\left(\omega_{1}-\omega_{2}, k_{1}-k_{2}\right),\left(2 \omega_{1}, 2 k_{1}\right)$ and $\left(2 \omega_{2}, 2 k_{2}\right)$ which are the sum and difference combinational harmonics respectively [18] along with harmonics of individual fundamental wave. Nonlinear acoustic parameter can be expressed as $4 A_{\omega_{1}+\omega_{2}} / A B k_{1} k_{2} x$ [15]. In this work, the amplitude of the sum frequency component has been evaluated for 04 various positions for varying propagation distances to assess the localized deformation and is denoted as $\mathrm{A}_{\omega 1+\omega 2}$.

\subsection{Selection of fundamental frequencies}

Fig. 1 shows the phase velocity and group velocity dispersion curves of Lamb waves for $2 \mathrm{~mm}$ thick modified 9Cr-1 Mo steel plate. Due to multimodal and dispersive nature of Lamb waves, fundamental mode is desirable for any NDE application [15]. Accordingly, in this work, S0 mode was chosen due to its almost non-dispersive nature and uniform mode shape through-out the thickness in lower frequency regime. Fig. 2 shows the frequency response of the undamaged sample during excitation by the frequency starting from $0.28 \mathrm{MHz}$ to $0.91 \mathrm{MHz}$ based on the encircled region as shown in Fig. 1. $0.41 \mathrm{MHz}$ and $0.73 \mathrm{MHz}$ have been chosen for this study for getting the resonance condition as these two frequencies give almost equal amplitude responses; although $0.61 \mathrm{MHz}$ gives maximum amplitude, but it is not used because it may suppress the other frequency components. Moreover, the difference of group velocity is more between the selected frequency components as it'll help to do the spatial scanning more reasonably (see Table 1).

\section{Finite element simulation for lamb wave mixing}

\subsection{Finite element model}

Propagation of Lamb wave and mixing were simulated using simulation software COMSOL Multiphysics. A $2 \mathrm{~mm}$ thick steel plate was taken for finite element simulation in two-dimensional domain considering plane strain condition. Degraded material properties were given in a localized region on the plate assuming the region is hyperelastic. Murnaghan material model was adopted for this degraded region where strain energy function was defined with respect to Lame's constants $(\lambda$

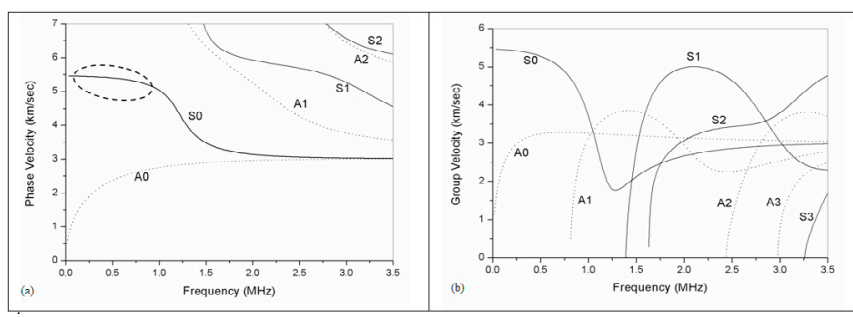

Fig. 1. Dispersion curves for $2 \mathrm{~mm}$ modified 9Cr.-1Mo steel; (a) Phase velocity, (b) Group velocity. 


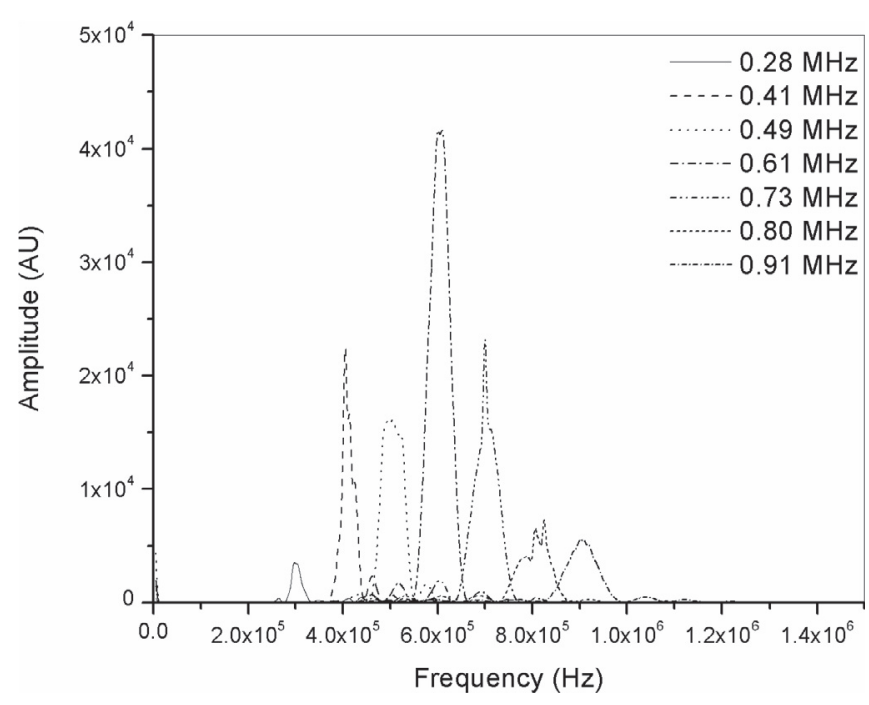

Fig. 2. Frequency response of the as-received sample.

Table 1

Shows the phase velocities and group velocities of the selected two fundamental frequencies of S0 mode.

\begin{tabular}{lll}
\hline Frequency & Phase velocity $\left(\mathrm{C}_{\mathrm{p}}\right)$ & Group velocity $\left(\mathrm{C}_{\mathrm{g}}\right)$ \\
\hline $0.41 \mathrm{MHz}$ & $5422 \mathrm{~m} / \mathrm{sec}$ & $5345 \mathrm{~m} / \mathrm{sec}$ \\
$0.73 \mathrm{MHz}$ & $5314 \mathrm{~m} / \mathrm{sec}$ & $4938 \mathrm{~m} / \mathrm{sec}$ \\
\hline
\end{tabular}

and $\mu)$ and higher order elastic constants $(l, m \& n)$ [17] to represent the microstructural degradation or nonlinearity in the material. Rest of the portion in the plate was given linear material properties. Two hanning windowed 05 cycle time domain tone burst signals of frequencies 0.73 and $0.41 \mathrm{MHz}$ were applied as pressure transient loading on one face of the plate in positive x-direction as shown in Fig. 3. Table 2 shows the material properties that were used for simulation.

The denser mesh, higher order element and smaller time step are desirable for accurate simulation result; but the calculation time will be very high and it will cost more in terms of computer resources. So, optimum mesh size and time step were chosen for this study in order to obtain high accuracy and efficiency. The sizes of the elements were chosen in such a manner that the desired propagating as well as generated frequency could be spatially resolved. Accordingly, as recommended in Ref. [19], 20 nodes per wave length were taken for the element size $\left(l_{e}\right)$ and 20 points per cycle of the highest frequency component were taken for effective temporal resolution $(\Delta t)$.

$l_{e}=\frac{\lambda_{\min }}{20}$ and $\Delta t=\frac{1}{20 f_{\max }}$

The various parameters used for finite element simulation are shown in Table 3.

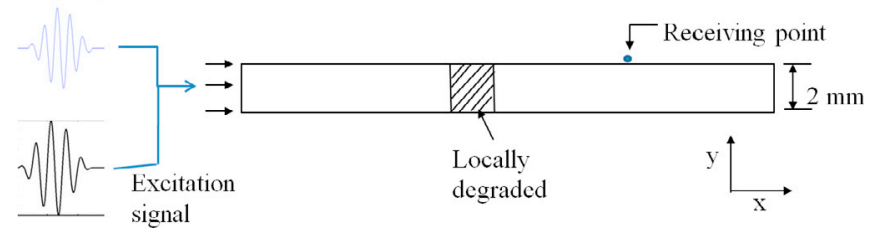

Fig. 3. Schematic of the plate with localized degradation for finite element simulation.
Table 2

(Material properties used for simulation).

\begin{tabular}{ll}
\hline$\nu$ (Poisson's ratio) & 0.31 \\
\hline$\rho\left(\mathrm{kg} / \mathrm{m}^{3}\right)$ & 7850 \\
$\mathrm{E}(\mathrm{Pa})$ & $2.1 \times 10^{11}$ \\
$\lambda(\mathrm{Pa})$ & $1.15 \times 10^{11}$ \\
$\mu(\mathrm{Pa})$ & $7.69 \times 10^{10}$ \\
$l(\mathrm{~Pa})$ & $-3 \times 10^{11}$ \\
$\mathrm{~m}(\mathrm{~Pa})$ & $-6.2 \times 10^{11}$ \\
$\mathrm{n}(\mathrm{Pa})$ & $-7.2 \times 10^{11}$ \\
\hline
\end{tabular}

Table 3

(Parameters used for finite element simulation).

\begin{tabular}{ll}
\hline$\omega_{1}(\mathrm{MHz})$ & 0.41 \\
\hline$\omega_{2}(\mathrm{MHz})$ & 0.73 \\
$\omega_{1}+\omega_{2}(\mathrm{MHz})$ & 1.14 \\
$\mathrm{l}_{\mathrm{e}}(\mathrm{mm})$ & 0.23 \\
$\Delta \mathrm{t}(\mathrm{sec})$ & $4.4 \times 10^{-8}$ \\
\hline
\end{tabular}

\subsection{Simulation results}

\subsubsection{Study-I}

In this study, mixing of two selected Lamb wave modes was allowed to occur at degraded region as well as at linearly elastic region (nondegraded) to see the generation of sum/difference frequency components in presence of nonlinearity within the material. Fig. 4 (a) shows the applied signal that consists of two selected fundamental frequencies of S0 mode and the fft of the triggered signal [Fig. 4 (b)] shows the presence of 0.41 and $0.73 \mathrm{MHz}$. The time delay was given to $0.41 \mathrm{MHz}$ signal (based on the group velocities of the two signals) such that the mixing could occur at the desired localized degraded region (Fig. 3). The simulation was performed such that the interaction of the excitation waves occurs at the degraded region and also away from degraded region. Fig. 5(I-(a)) shows the simulated received signal and corresponding fft [Fig. 5 (I- (a))] when interaction occurs at degraded zone. The generation of sum frequency $\left(\omega_{1}+\omega_{2}=1.14 \mathrm{MHz}\right)$ component can be seen in Fig. 5 (I- (a)) due to the interaction of the fundamental waves in presence of nonlinearity of the degraded region; but no side-bands (sum or difference frequency component) are observed [Fig. 5 (II- (a) \& (b))] in case of interaction at non-degraded i.e. linearly elastic region. Amplitude of this third wave depends on the degree of degradation and so this technique could be used for assessing the localized microstructural degradation due to damage.

\subsubsection{Study- II}

In this study, mixing of two selected fundamental Lamb wave modes was allowed to occur at 04 different positions as shown in Fig. 6 to evaluate the mode shapes of the generated mixed signals. The four mixing positions on the sample were modeled using Murnaghan's nonlinear elastic constants as given in Table 2 . The points of applying the pressure load as well as the receiving point were kept constant; but time-

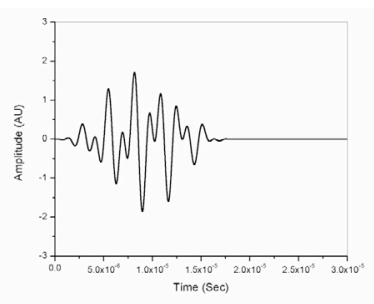

(a)

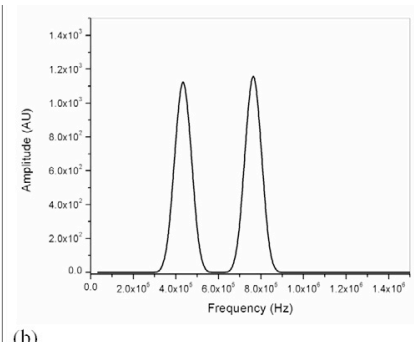

(b)
Fig. 4. (a) Excitation signal, (b) fft of the excitation signal showing the presence of $0.41 \mathrm{MHz}$ and $0.73 \mathrm{MHz}$. 


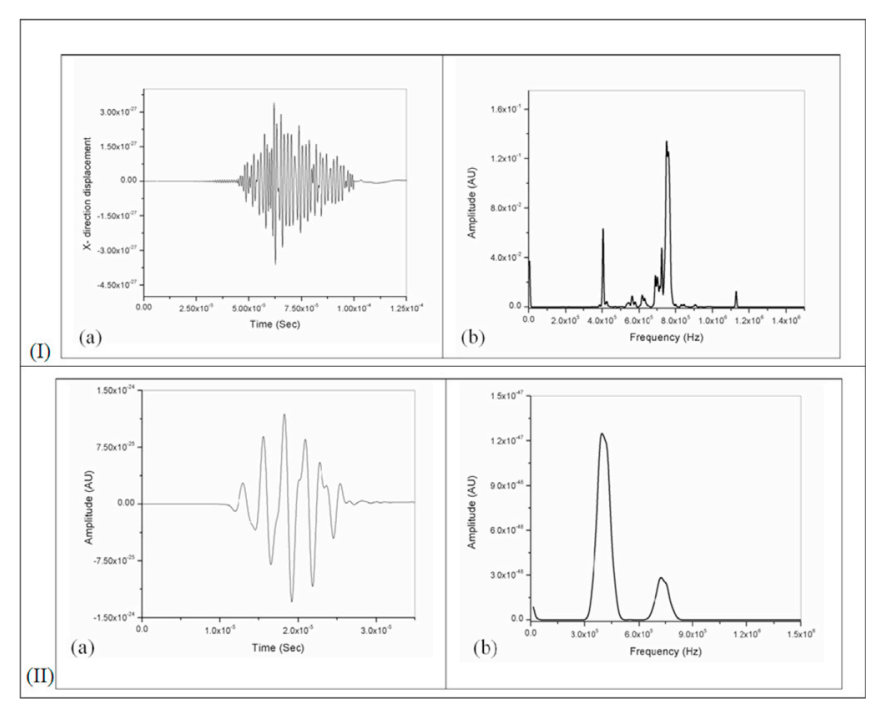

Fig. 5. [I- (a)] Received signal in presence of nonlinearity, [I- (b)] fft of the received signal showing the presence of side-bands of the primary fundamental waves; [II-(a)] Received signal in absence of nonlinearity, [II-(b)] $\mathrm{fft}$ of the received signal showing the absence of side bands.

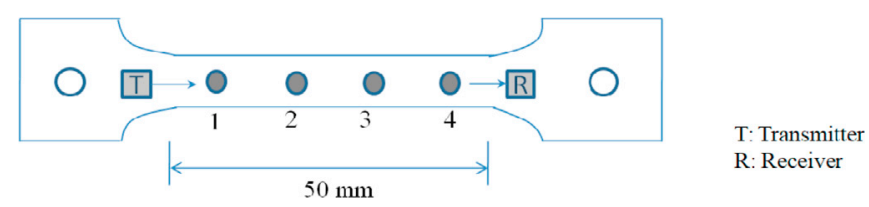

Fig. 6. Schematic of the sample showing 04 mixing positions along the gage length.

delay was given to one of the fundamental waves according to the group velocities of the waves and mixing point positions. All interaction points were separated by $10 \mathrm{~mm}$ from each other. Two fundamental signals of $0.41 \mathrm{MHz} \& 0.73 \mathrm{MHz}$ along with the received mixing signals from 04 different positions have been shown in Fig. 7. Time delays given to one of the fundamental signals have also been mentioned in the figure. The profiles of the mode shapes as can be seen in Fig. 8, show that the udisplacements (x-component) are approximately constant through the thickness indicating that the damage evaluation can be done through-out the cross section of the plate, whereas v-displacements (y-component) are varying along the thickness; but both the mode shapes are symmetric in nature.

\section{Experimental}

\subsection{Experimental procedure}

A high power ultrasonic pulser RAM 5000 from RITEC Inc was used for generating high voltage tone burst signals of 5 cycles at exciting frequencies of $0.71 \mathrm{MHz}$ and $0.43 \mathrm{MHz}$. Two commercial piezoelectric transducers of central frequencies of $1 \mathrm{MHz}$ were used for generating and receiving the signals. Fig. 9 and 10 show the experimental set-up for the nonlinear Lamb wave mixing experiment and the frequency response of the transducer respectively. One transducer was fixed to perspex-made wedge of angle $28^{\circ}$ to generate the desired frequency components and the other one was directly fixed to the surface of the sample to detect the mixing signal. Light lubrication oil was used for coupling the transducers with the wedge as well as with the sample surface.

A flat dog-bone specimen made of modified $9 \mathrm{Cr}-1$ Mo steel of thickness $2 \mathrm{~mm}$ was used for the creep experiment and Lamb wave mixing. A schematic of the specimen used for creep as well as Lamb wave

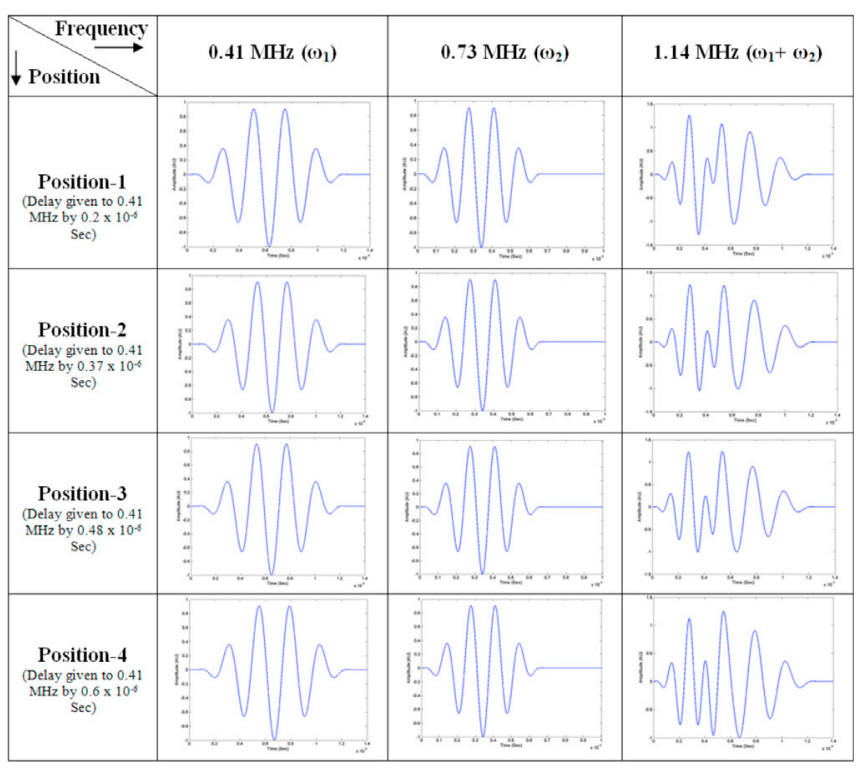

Fig. 7. Excitation signals and received signals for 04 different positions including time-delay.
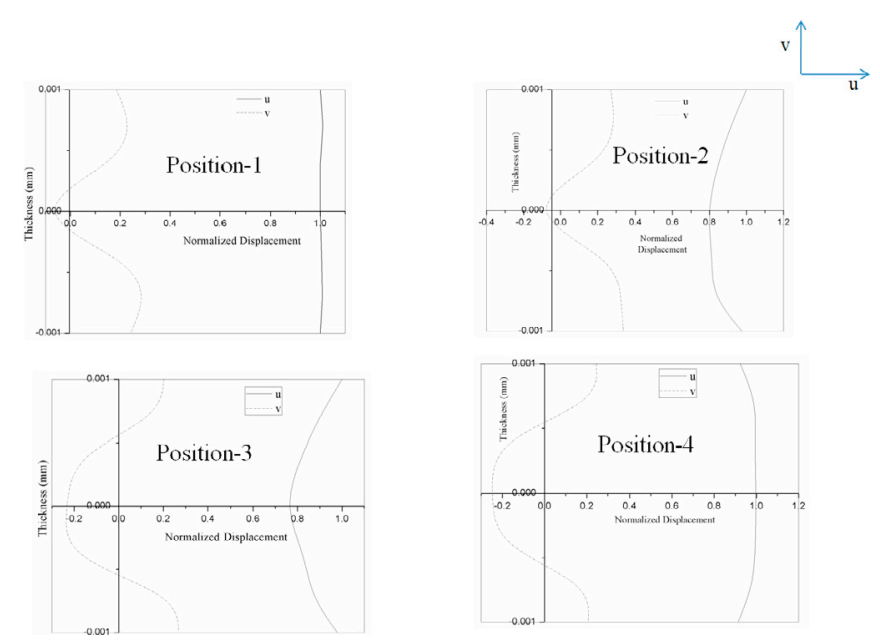

Fig. 8. Evolution of mode shape for each mixing position.

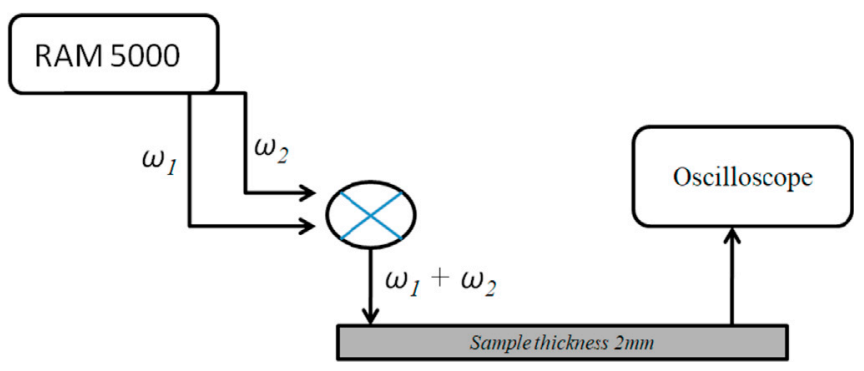

Fig. 9. Schematic of the experimental set-up.

mixing study is shown in Fig. 6. As can be seen, four different positions were taken with $10 \mathrm{~mm}$ separation with each other for mixing the Lamb waves to evaluate the localized plastic deformation during creep. Creep testing was done at $100 \mathrm{MPa}$ stress and $650^{\circ} \mathrm{C}$ temperature in constant load creep testing machine (make: MAYES; model: TC-30) with a lever arm ratio of 1:15. Interruption was done at various time intervals to evaluate the amplitude of the sum frequency component, $A_{\omega 1+\omega 2}$ for the 


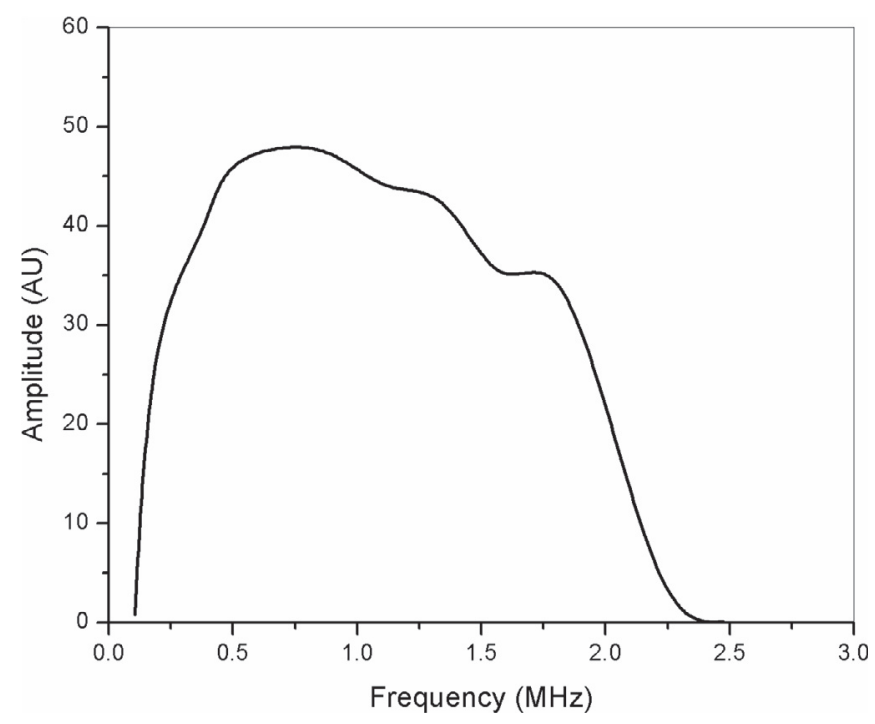

Fig. 10. Frequency response of the transmitter as well as receiver.

four defined positions on the gage length of the sample.

\subsection{Results \& discussions}

Two sets of experiments were done for this study. In the first set, the interruption was done at $10 \mathrm{hs}, 15 \mathrm{hs}, 20 \mathrm{hs}, 50 \mathrm{hs}, 100 \mathrm{hs}, 200 \mathrm{hs}$, and 300 hs. The sample failed at $396 \mathrm{~h}$. Fig. 11 shows the variation of normalized $A_{\omega 1+\omega 2}$ at the 04 positions along the gage length after every interruptions. The corresponding strain at each interruption has also been shown in this figure. Normalized $\mathrm{A}_{\omega 1+\omega 2}\left[\mathrm{~N}\left(\mathrm{~A}_{\omega 1+\omega 2}\right)\right]$ was defined as the ratio of the value of $\mathrm{A}_{\omega 1+\omega 2}$ at each interruption time to the highest value of $A_{\omega 1+\omega 2}$ obtained during all interruptions. It is seen that there is not much change in $\mathrm{N}\left(\mathrm{A}_{\omega 1+\omega 2}\right)$ till $50 \mathrm{~h}$; slight increment has been started at $100 \mathrm{~h}$ that corresponds to $1.3 \%$ creep strain and thereafter there is a rapid increase in $\mathrm{N}\left(\mathrm{A}_{\omega 1+\omega 2}\right)$ after $100 \mathrm{~h}$. Finally, the sample failed at the location (between position-1 \& 2) where this amplitude of the sum frequency component that represents the material degradation (so

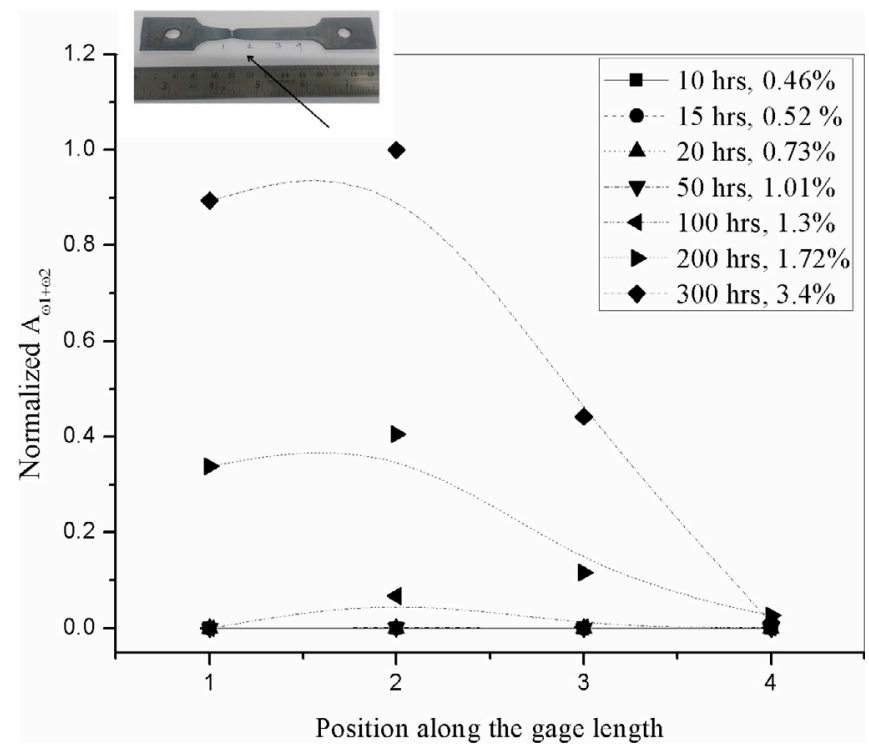

Fig. 11. First set: Variation of normalized $A_{\omega 1+\omega 2}$ (evaluated from wave mixing) along the gage length with interruption time and corresponding creep strain; inset: a view of the failed sample after $396 \mathrm{~h}$. increased nonlinearities) as damage progresses, was increased substantially.

In the second set of experiment, the number of creep interruption was increased to check the behavior of the variation of $\mathrm{N}\left(\mathrm{A}_{\omega 1+\omega 2}\right)$ along the gage length. Accordingly, the interruptions were at $6 \mathrm{~h} \mathrm{~s}, 10 \mathrm{~h}, 15 \mathrm{~h}, 20 \mathrm{~h}$, $27 \mathrm{~h}, 50 \mathrm{~h}, 76 \mathrm{~h}, 100 \mathrm{~h}, 200 \mathrm{~h}, 300 \mathrm{~h}$, and $400 \mathrm{~h}$; the sample failed at $440.5 \mathrm{~h}$. Fig. 12 displays the variation of normalized $\mathrm{A}_{\omega 1+\omega 2}$ at the 04 defined positions with time and creep strain. Here also, it is seen that initially there is very little change in $N\left(\mathrm{~A}_{\omega 1+\omega 2}\right)$ till $100 \mathrm{~h}$; increase in $\mathrm{N}$ $\left(\mathrm{A}_{\omega 1+\omega 2}\right)$ started from $200 \mathrm{~h}$ with a creep strain of $1.8 \%$ and drastically increased further at the position between $2 \& 3$. It also follows the same trend as the first set where the sample fails at the position where maximum $\mathrm{A}_{\omega 1+\omega 2}$ obtained much prior to its failure. Fig.13 shows the creep curves and curves of creep strain rate for both set-I \& II. In all these cases it can be seen that the value of $N\left(\mathrm{~A}_{\omega 1+\omega 2}\right)$ drastically increases approximately after $45-50 \%$ of the damage with a corresponding creep strain of approximately $1.8 \%$. Evolution of $1.14 \mathrm{MHz}\left(\omega_{1}+\omega_{2}\right)$ frequency component with creep interruption time at position- 3 of the second set is

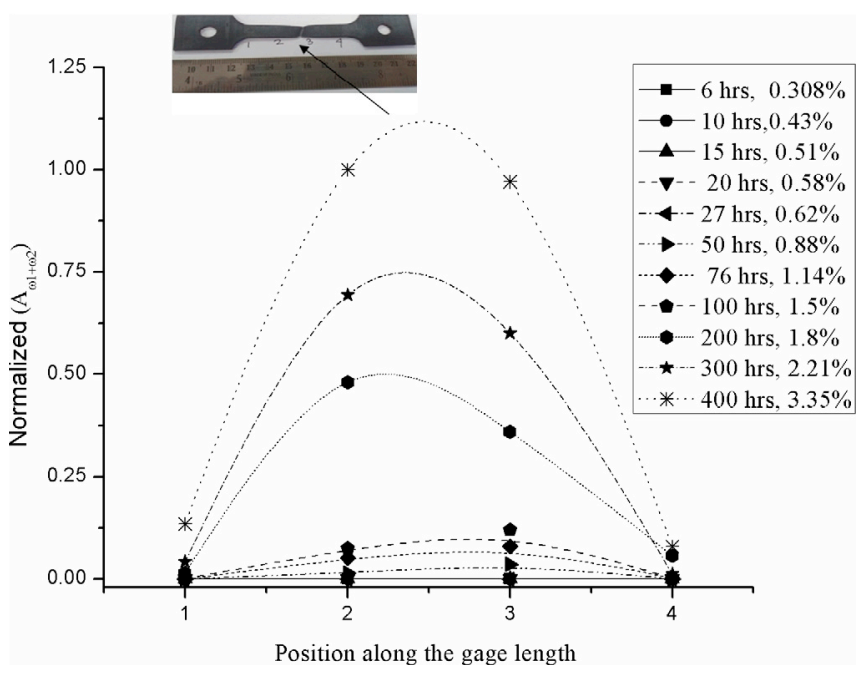

Fig. 12. Second set: Variation of normalized $A_{\omega 1+\omega 2}$ (evaluated from wave mixing) along the gage length with interruption time and corresponding creep strain; inset: a view of the failed sample after $440.5 \mathrm{~h}$.

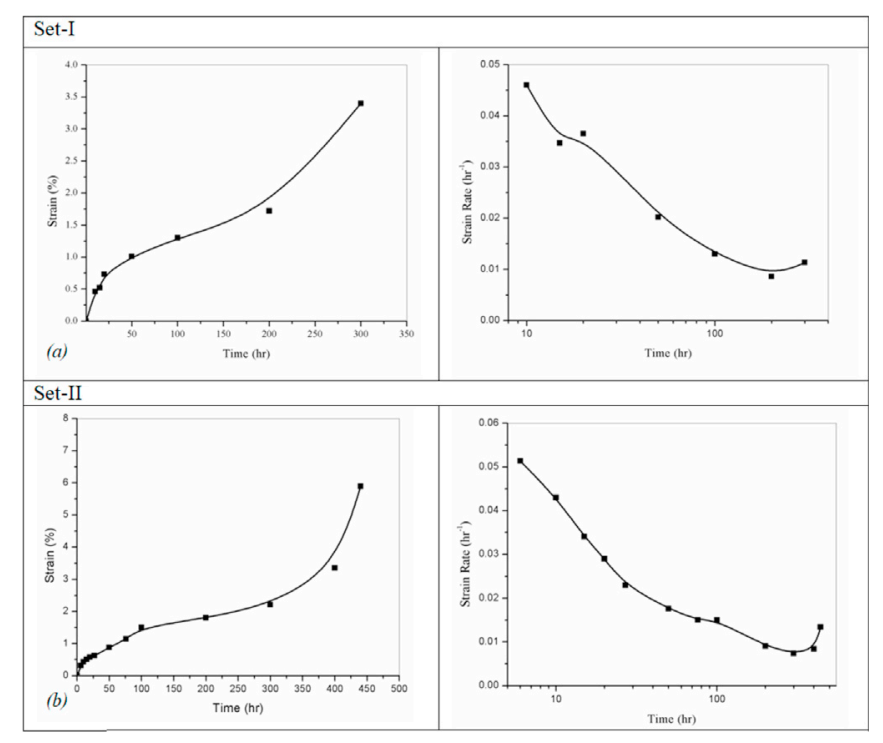

Fig. 13. Creep curves and creep strain rate variation for both the sets of samples. 


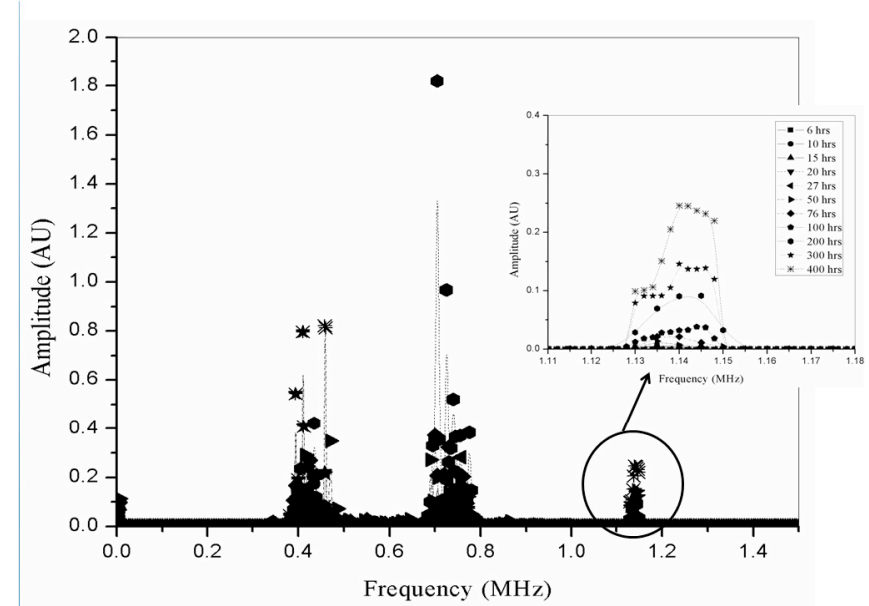

Fig. 14. Evolution of sum frequency component $(1.14 \mathrm{MHz})$ at various creep interruption time of position-3.

shown in Fig-14. Nonlinear ultrasonic technique enables to characterize the onset of plastic damage during any type of deformation process much earlier than the sample fails. If this plastic deformation is non-zero, then finite deformation theory introduces third order elastic constants that define the nonlinear Hooke's law (nonlinear stress-strain behavior). These third order elastic constants depend on the dislocation accumulation/behavior within an isotropic material during damage. Nonlinearity in the Hooke's law arises due to anharmonicity of the lattice and due to the dislocation displacement. Hikata et al. [20] derived the expression of the amplitude of harmonic component (A) due to contribution from dislocations. They showed that A is related to elastic constants of the materials and dislocations morphologies, neglecting attenuation of the fundamental and harmonic waves, by

$A=\frac{1}{4}\left[\frac{E_{2}}{E_{1}}+\frac{12}{5} \frac{\Omega \Lambda E_{1}^{2} L^{4} R^{3}}{\mu^{3} b^{2}} \sigma\right] \cdot\left(A_{1} k\right)^{2} \cdot x$

where, $E_{1}$ and $E_{2}$ are the appropriate moduli of the second and third order respectively. $\Omega=$ conversion factor from shear strain to longitudinal strain; $2 \mathrm{~L}=$ dislocation loop length; $\mathrm{R}=$ resolving shear factor; $\mu=$ shear modulus; $\mathrm{b}=$ Burgers' vector; $\sigma=$ applied small longitudinal stress; $\mathrm{A}_{1}=$ amplitude of the fundamental wave; $k=$ wave vector and $x=$ wave propagation distance. $E_{2} / E_{1}$ is the amplitude due to the lattice anharmonicity and $\frac{12}{5} \frac{\Omega \Lambda E_{1}^{2} L^{4} R^{3}}{\mu^{3} b^{2}} . \sigma$ represents the amplitude due to dislocation displacements. During the progress of creep damage, localized variation in dislocation evolution within the material introduces the change in third order elastic constants and increased material nonlinearities locally.

As the mixing of two primary fundamental waves generates a third new wave related to the sum frequency of fundamental waves in the presence of nonlinear interaction within the material due to dislocation evolutions, this Lamb wave mixing could be the potential technique for localized damage detection.

\section{Conclusions}

- Nonlinear Lamb wave mixing using nonlinear ultrasonic technique was done to assess the localized deformation during creep.

- Generation of sum frequency component of the fundamental primary waves was investigated in finite element simulation in presence of third order elastic constants that represent the material non-linearity in a pristine homogeneous plate.

- Experimentally it was seen that onset localized deformation could be predicted much prior to its failure.

- Increase in amplitude of the sum frequency component was started after $45-50 \%$ damage of the sample.

\section{Acknowledgement}

The authors are thankful to the Director, CSIR- NML for providing the infrastructural and financial support to carry out the work. Help rendered by Dr. J. Swaminathan during creep experiment is also gratefully acknowledged.

\section{References}

[1] Chillara VK, Lissenden CJ. Review of nonlinear ultrasonic guided wave nondestructive evaluation: theory, numerics, and experiments. Opt Eng 2016;55(1). $011002(1-15)$

[2] Liu M, Tang G, Jacobs LJ, Qu J. Measuring acoustic nonlinearity parameter using collinear wave mixing. J Appl Phys 2012;112. 024908 (1-6).

[3] Goldberg ZA. Interaction of plane longitudinal and transverse elastic waves. Akust Zh 1960;6(3):307-10.

[4] Jones GL, Kobett DR. Interaction of elastic waves in an isotropic solid. The Journal of J. Acoust. Soc. Am. 1963;35(1):5-10.

[5] Taylor LH, Rollins Jr FR. Ultrasonic study of three-phonon interactions. I. Theory. Phys Rev 1964;136(3A):A591-6.

[6] Rollins Jr FR, Taylor LH, Todd Jr PH. "Ultrasonic study of three phonon interactions. II. Experimental Results. Phys Rev 1964;136(3A):A597-601.

[7] Krasilnikov VA, Zarembo LK. Nonlinear interaction of elastic waves in solids. IEEE Trans Son Ultrason 1967;14(1):12-7.

[8] Holt AC, Ford J. Theory of ultrasonic three-phonon interactions in single- crystal solids. J Appl Phys 1969;40(1):142-8.

[9] croxford AJ, Wilcox PD, Drinkwater BW, Nagy PB. The use of non-collinear mixing for nonlinear ultrasonic detection of plasticity and fatigue. J Acoust Soc Am 2009; 126(5):EL117-122.

[10] Demcenko A, Akkerman R, nagy PB, Loendersloot R. Non-collinear wave mixing for non-linear ultrasonic detection of physical ageing in PVC. NDT Int 2012;49:34-9.

[11] Jiao J, Sun J, li N, Song G, Wu B, He C. Micro-crack detection using a collinear wave mixing technique. NDT Int 2014;62:122-9.

[12] Jingpin J, Junjun S, Guanghai L, Bin W, Cunfu H. Evaluation of the intergranular corrosion in austenitic stainless steel using collinear wave mixing method. NDT Int 2015;69:1-8.

[13] Lee DJ, Cho Y, Li W. A feasibility study for Lamb wave mixing nonlinear technique. In: 40th ann. Rev. Of prog. In quant. NDE, AIP conf. Proc, vol 1581; 2014. p. 662-6.

[14] Lissenden CJ, Liu Y, Chillara VK, Choi G, Cho H. Nonlinear guided wave mixing for localized material state characterization. Physics Procedia 2015;70:668-71.

[15] Jingpin J, Xiangji M, Cunfu H, Bin W. Nonlinear Lamb wave-mixing technique for micro-crack detection in plates. NDT E Int 2017;85:63-71.

[16] Rose JL. Ultrasonic waves in solid media. New York: Cambridge University Press; 1999.

[17] Murnaghan FD. Finite deformation of an elastic solid. New York: John Wiley \& Sons; 1951.

[18] Hasanian M, Lissenden CJ. Second order harmonic guided wave mutual interactions in plate: vector analysis, numerical simulation, and experimental results. J Appl Phys 2017;122. 0884901 (1-13).

[19] Moser F, Jacobs LJ, Qu J. Modeling elastic wave propagation in waveguides with the finite element method. NDT E Int 1999;32:225-34.

[20] Hikata A, Chick BB, Elbaum C. Dislocation contribution to the second harmonic generation of ultrasonic waves. J Appl Phys 1965;36(1):229-36. 